\title{
OCCLUSION OF TEETH IN RELATION TO THE TEMPORO- MANDIBULAR ARTICULATION
}

\author{
By Richard Summa, D.D.S. Kansas City, Mo.
}

"T

HE more successful plan is to move the mandibular teeth forward and leave the condyle in the anatomic position."

"Now, what was demanded in those cases was the forward movement of the mandibular arch with the condyle remaining in the anatomic position."

"By moving the teeth forward according to the above method we are positive that the mandibular arch has been lengthened without the condyle being moved forward."

These quotations are taken from an editorial in the May, 1921, International Journal of Orthodontia and Oral Surgery. Every one who has attempted to correct malocclusions involving a correction of the mesio-distal relation of the molars (which in its finality means a correction of the mesial distal relation of the maxillary and mandibular arches) has met more or less disappointment, usually more.

The dissections and study of the temporo-mandibular articulation conducted by Professor H. J. Prentiss and the writer confirmed the rational assumption that, not only the loss of teeth, but also the occlusion of teeth is recorded in this joint.

Many years ago while this problem was being discussed, a writer used the appropriate phrases of "jumping the bite" and "jumping the occiusion." The former was applicable to an attempt to move the mandibular arch forward as a whole, thus the only change was expected to take place in the temporomandibular articulation; the latter, jumping the occlusion, is applicable to moving individual teeth as referred to in the editorial under consideration.

If the observations of Professor Prentiss and the writer are correct, then every permanent change in the position of teeth is recorded in the temporomandibular articulation.

There is no doubt that jumping the occlusion is the logical procedure for various reasons, but it is likewise logical to assume that an adjustment of the temporo-mandibular articulation is imperative and does take place in the cases where the teeth remain in the corrected positions.

If the nature of the tissues composing this articulation is taken into account, it is not surprising that its adaptation to changes in the occlusion of teeth is very gradual.

While we have no data on hand to make any positive assertion as to the corresponding depth or shallowness of the glenoid fossa in various types of malocclusion, the writer will hazard the opinion that in distal mandibular occlusions, a shallow glenoid fossa and rather flat condyle will prevail. To retain teeth which have been changed from a Class II, Division 1, malocclusion into a normal mesio-distal occlusion, a deeper glenoid fossa and corresponding condyle will be required.

Does this change take place? 